\title{
Traditional Macro-tower to Heterogeneous Cellular Networks: A Survey
}

\author{
Janmoni Borah $^{1 *}$ and Joyatri Bora ${ }^{2}$ \\ NERIST Deemed University, Arunachal Pradesh, India \\ 1borah1989@gmail.com, ${ }^{2} j b @ n e r i s t . a c . i n$
}

\begin{abstract}
Cellular operators are moving towards deployment of heterogeneous cellular networks (HetCNs) not just to deliver services to remote locations but also to spread out data capacity in crowded urban areas. HetCNs, along with traditional macro towers may also include relays, smaller base station and distributed antennas. These new infra with small base stations and cells (e.g., metrocells, microcells, picocells, and femtocells) have advantages such as smartness, cheap, less obstructive, and easier installation compared to traditional homogeneous macrocell networks. Traditional single towers along with these small cells can provide services not only to outside busy intersections but also to residential homes and offices. This paper addresses some of the major concerns of today's cellular system such as coverage, capacity, interference and spectrum reuse. The paper also presents and discusses advantages and future needs of denser deployment of base stations and cells in a heterogeneous cellular network. Literature survey on some of the recent research works associated with spectrum allocation and interference management are presented. Major issues such as user association, off-loading of users from a macro to small cells etc, and potentials of superimposing small BSs with traditional macro cell BS are discussed.
\end{abstract}

Keywords: Base station, HetCN, ICI mitigation, Resource allocation, Small cells, User demands

\section{Introduction}

Coverage, Capacity, Interference and Spectrum reuse are vital concerns of cellular systems. Cellular or mobile networks influence our daily life, as fast communication with low cost lies in our nature. It extends over a large geographic area that is dividing into cells. All the cells in the network offer radio coverage to a wide geographic area, such as city, state or nation. Cellular network enables numerable portable devices to communicate among them as well as with fixed-line telephone. A fixed transceiver node named Base Station (BS) that are linked to a core network of telecommunication vendors and the internet, serves mobile users within the confined region of each cell in the network. The available frequency spectrum and the number of channels that can be supported by the available bandwidth in cellular network manage the number of simultaneous calls. Cellular networks achieve a higher capacity and serving larger areas by adding additional BSs is its advantage as mobile user need less power for transmission when BS are in close vicinity [1]. Earlier some classical approaches and schemes were considered for allocating resources such as Frequency reuse, FDMA, TDMA and CDMA [3]. Today's major breakthrough and promising approaches includes OFDMA, MIMO techniques, Cellsectorization, Fractional Frequency reuse, BS-coordination, User scheduling, BScooperation (Network MIMO) and Massive MIMO [2].

Received (August 29, 2017), Review Result (November 13, 2017), Accepted (November 15, 2017)

* Corresponding Author 
Nowadays, most of cellular operators and vendors are leading towards deployment of heterogeneous cellular networks (HetCNs) not just to provide services to remote areas rather to enlarge data capacity in urban areas having congestion. HetCN may include divided small cells, relays, and smaller base stations (BSs) with distributed antennas. These new infra with miniaturized BSs and cells (e.g., metrocells, microcells, picocells, and femtocells) are cheap, smart, less obstructive, and easy to install compared to traditional homogeneous macrocell networks. Traditional towers along with small cells within local range can provide services not only to outside busy intersections but also to residential homes and offices. HetCNs thus fetch the network to the vicinity of users and thereby drastically enhance the overall network capacity and a superior spatial resource reuse is attained. Due to such striking features and pros, in the last few years HetCNs have attain attention largely in wireless industry and research. In this paper, we present a summary of research works being carried out by researchers and wireless communication engineers in cellular network areas. The rest of the paper is structured as follows. In Section II, some advantages and future need of heterogeneous cellular network over homogenous one are presented and discussed. Literature survey on some major areas and recent research contributions are summarized in Section III. Some relevant issues and potentials of deploying small cells along with macro cells are discussed in Section IV. Lastly, Section V draws the conclusion part of this survey.

\section{Homogeneous to Heterogeneous Cellular Network}

In the last few years, traditional homogeneous $2 \mathrm{G}$ and $3 \mathrm{G}$ cellular networks were usually managed by macro BSs that provides blanket coverage within its range. However, indoor users suffer due to low signal quality because of strong penetration and high path loss. HetCN improves coverage for indoor areas by allowing flexible deployment or installation of low power and low complexity micro, pico and femto BSs. Typically, HetCN comprises both traditional macro-BS with high transmit power for cell-edge coverage and other micro, pico, and femto BSs depends on network demands with low transmit power for cell-centre users.

Due to limited spectrum and considerable increase in the number of users, today's cellular networks can no longer meet the user demands such as coverage and throughput. Moreover, most of the resource management and ICI mitigation techniques found in literature are more or less considers homogeneous macrocell scenarios, and thus unable to meet the expectation of densely deployed heterogeneous one. The grounds behind is that, the dominant interferers are limited in homogeneous as compared to dense heterogeneous networks. In addition, the never ending hunger for higher data rates and need for a more efficient use of the limitedly available radio spectrum has led to development of new technologies. Unfortunately, although densely deployed small cells is a better approach for wireless network, but also will brought new challenges for engineers such as efficient resource allocation, interference management etc.

\section{Literature Survey on Recent Works}

Recently, Resource allocation and Interference management (especially Inter-Cell Interference (ICI) for cell-edge users) have become major concerns for communication engineers, as because an effective management of ICI and available spectrum can improve cell-edge signal quality. ICI is always been a major degrading factor in terms of cell-edge user throughput. ICI in cellular networks occurs mainly due to use of same radio frequency in the neighboring cells. ICI problem has been broadly discussed in text: from homogeneous networks to dense femtocell networks. Most widely used ICI mitigation techniques and approaches include Frequency Reuse (FR), Fractional Frequency Reuse (FFR), Cell Sectorization, BS-coordination and BS-cooperation [2]. 


\subsection{Homogeneous Cellular Networks}

By homogeneous cellular networks, it refers to network with same size and each having centralized BSs among each cell. These networks mainly based on noncooperative spatial reuse partitioning approach to combat the problems of fading as well as interference. Several macro-BSs are placed in the entire area of service, more or less evenly distributed to form a cellular structure. Mobile users close to or in the range of home BS receive good SNR. While in case, users are in coverage range of multiple BSs, assigning orthogonal resources with FDMA, TDMA or CDMA reduces the interferences. Accordingly, the interferences are controlled by choosing high reuse factor (e.g. 3, 4, 7, 9 and 12) and fixing the BSs transmits power. The reuse approach indeed reduced the interference, but at cost of reduced resources in each cell. Consequently, interference and reuse limit the spectral efficiency $(\mathrm{bps} / \mathrm{Hz})$.

Spectrum reuse schemes such as FR and FFR have been extensively used in cellular system in order to extend the geographic coverage area and primarily for capacity enhancement, interference reduction. FR scheme avoids utilization of same frequency in the neighboring cells to reduce interference, while FFR scheme allows FR within a cell such that for cell-centre users assigns lower FR (FR-1) and cell-edge ones with higher reuse by employing sectors in the cell [4], [8]. MIMO techniques refer to use of multiple antennas at transceiver. In cellular network, it allows a transceiver to encompass multiple antennas that spatially multiplex channels among single or multiple users and thus increases the throughput by spectral reuse. Sectorization allows partitioning of cell radially into multiple sectors and reuse of resources in each sector, which enhances the spectral efficiency of each cell. In BS-coordination, multiple neighboring cells are allows to acquire the Channel State Information (CSI) of cell-edge users and thus allows to coordinate for the data transmission in a way such that one home cell is selected among these cells for transmission and other cells will engaged in designing precoding matrix by considering the intended user. The approach is also known as inter-cell coordination strategy as it pre-cancels the interferences at all neighboring cell and one cell among coordination is selected for transmitting information. In addition, the resources of the coordinating cells are made accessible to both cell centered and edge users and thereby mitigate interferences [4]. In Network MIMO, the concept of serving an intended user by an individual BS in a cell vanishes. The scheme allows connection between several BS antennas belonging to different cells using high-capacity backhaul link to serve the user. BSs via this link network share not just the CSI, moreover data signal of intended user. Hence, combined use of multiple BSs of different cells mitigates the ICI problem [8]. In spite of ICI mitigation and capacity enhancements, network vendors are still struggling to convince the increasing users and its demands. An efficient and optimum allocation or sharing of resources among huge number of users is of great concern. In such scenario, scheduling not only helps in allocation of resource among users but also decide when and which user should transmit or receive using that resources.

In [4], the capacity and cell-edge performance based on distance from BS is presented, by considering frequency reuse schemes and a coordination strategy among cells. In [5], FFR with sectorization and coordinated fair scheduling scheme is used to improve the cell edge users and to maintain fairness in resource allocation among users. Rizwan Ghaffar et al., [6], have investigated the downlink performance of cell-edge users by using propose interference suppression strategy and FFR scheme of 1.5 for edge users. In [7], a comparison is made between distributed and traditional centralized cellular system and cell-edge effects of both the systems is evaluated by considering both with or without ICI. Li-Chun Wang et al., in [8], have discussed a Network MIMO based 3-cell architecture by employing tri sector based regular and rearranged fractional frequency reuse schemes. Kianoush Hosseini et al., in [9], have made a comparison on two distinct interference mitigation techniques: Network MIMO and Large Scale-MIMO, for multicell downlink network. The results shows that user's in network MIMO system receives intended 
signals from multiple BSs whereas in LS-MIMO system user receives the same only from the closest BS. In [10], four major cooperation techniques for multi-cell networks: Interference coordination, MIMO cooperation, Rate-limited MIMO cooperation and Relay-assisted cooperation have been discussed. Yifan Liang et al., in [11], have made a comparison between two well-known base station approaches: Network MIMO and Densification of BS, which greatly helps in mitigating interference and system capacity enhancement. In [12], throughput performance of a downlink cellular network is compared for Single user, Multi-user with sectorization and Network MIMO with coordination among cells. In [13], it is presented that higher-order sectorization (more than 3 sectors) of cell provides not only better performance but also throughput scales directly with increase in number of sectors compared to conventional 3 sectors per cell. In [14], the performance analysis of a sectorized cellular system is presented by considering non-cooperative and cooperative transmission. Thomas Novlan et al., in [15], have provided a comparison on two main FFR deployment approaches: soft frequency reuse (SFR) and strict FFR for OFDMA cellular networks. In [16], a dynamic FFR scheme has been proposed which divides subcarriers into groups rather than users in a cell such that subcarriers allocation problem in OFDMA cellular networks can be overcome and users can access all the subcarriers within a cell. Abdelhalim Najjar et al., in [17], have addresses the cell-edge interference problem in multi-cell OFDMA system and presented an efficient frequency reuse scheme and threshold switching mechanism based on received user SINR and distance between user and BS to reduce it. Md. Assad in [18], has presented inter-cell interference analysis in multicellular OFDMA based system by employing FFR technique among cells. Qinghua Li et al., in [19], have provide an overview of some key MIMO techniques for uplink and downlink in WiMAX and LTE standards. In [20], some interference mitigation and cancellation techniques is presented which can be employed in 4G OFDM systems. In [21], co-channel interferences are analyzed for MIMO based multi-cell heterogeneous network and an algorithm is proposed which not only reduce the number of ICIs but also increase the system capacity. Ajay Thampi et al., in [22], have presented a 3-cell static clustered network and employs FFR technique for users in cluster-center and cluster-edge to mitigate interference on uplink network. In [23], Xin Yongchao et al., have addressed two systems namely: Three-node CCFD system and FFR based CCFD system, for suppression of Inter-Terminal and InterCell interferences in a CCFD cellular system. Yang Zhang et al., in [24], have proposed a Partial Interference Alignment (PIA) scheme to enhance the throughput. The proposed scheme requires relatively less amount of antennas and properly ignores some weak interference links in multi-cell networks. In [25], the problem associated with estimation of number of interferers from co-channel cell has been addressed for a MIMO cellular network. Vincenzo Sciancalepore et al., in [26], have addresses a pure scheme based on base station scheduling with frequency reuse 1 rather than frequently adopted user scheduling. Guanding $\mathrm{Yu}$ et al., in [27], addresses resource allocation problem and proposed a novel user-scheduling algorithm for cellular network with full-duplex BSs. Arash Asadi et al., in [28], provides a survey and taxonomy to opportunistic scheduling which were emerges in the last two decades. Authors in [29], proposed a mechanism in which neighboring nodes can notify each other about the resource utilization and thus nodes are avoid to utilize high transmission power in those areas. In [30] and [31], authors have used the concept of minimizing transmission power and address the user interference tolerance for efficient allocation and management of resources in traditional macrocell and densely deployed small cells.

\subsection{Heterogeneous Cellular Networks (HetCNs)}

In modern 4G cellular networks (e.g., LTE-A and WiMAX), size of cells are reducing and reuse factors are approaching to 1. Modern OFDM based cellular system uses flexible reuse pattern that changes dynamically according to user's distribution and traffic 
demands. The approach allocates full spectrum to users near BS and orthogonal frequencies for users in the cell edge [2]. HetCN offers some attractive features and advantages and can significantly boost the capacity of a network via spatial reuse. HetCN allows flexible deployment of low power and low complexity micro, pico and femto BSs. For huge number of users, HetCN may be bulid with traditional macro-BS with high transmit power for cell-edge coverage and pico, and femto BSs with low transmit power for cell-centre users. In HetCNs, macrocells provide service to high mobility users by covering a wide area, whereas densely deployed femto, pico and micro cells provide services to low mobility user within a cell. However, due to dense deployment of cells major complications and technical issues arises such as efficient allocation of resources and interference management.

Recent advances in HetCNs took place in following major research areas: User association, Resource allocation and interference management, new antenna techniques, Dynamic TDD, Coexistence of LTE and WiFi HetCNs, and Network topologies and cooperative communications. In [32], Yibing Li et al., have not only addresses major drawback associated with conventional static ICI-coordination strategies such as FFR, soft-FFR, but also proposes a universal FR scheme for heterogeneous network to achieve good throughput for all users by allowing them to use all spectrum in a cell and developed a sub-band allocation algorithm. In [33], Guogang et al., have proposed a novel Adaptive Frequency Reuse (AFR) scheme for ICI coordination in femtocell network. The proposed scheme allows division of all sub-channels (SCs) into primary SCs and secondary SCs, such that primary SCs maintain orthogonality among neighboring femtocells. Aysha Ebrahim et al., in [34], have discuss and propose a novel sleep mode (SL) based resource management in which switching off of certain small cells is done to mitigate interference and enhance the capacity of a heterogeneous networks. In [35], Binnan Zhuang et al., have proposed an optimized framework by jointly using approaches: spectrum allocation, user association, and cell activation. The authors also studied about possible activation of few small cells at an instance to preserve energy and for efficient utilization of resources in heterogeneous cellular networks. Arsalan Saeed et al., in [36], have investigate the downlink performance of a OFDMA based heterogeneous network by considering macrofemto inter- tier interference. The study addresses downlink resource allocation problem in HetCN, such that both macro and femtocells are transmitting on same frequency bands and nodes are allowed to use entire bandwidth within a cell. In [37], authors presented a resource partition and allocation scheme for two-tier downlink femtocell networks, where macrocells with FFR are superimpose with femtocells. Shahadate Rezvy et al., in [38], have proposed an efficient technique to improve the system capacity and to mitigate interferences associated with the existing macro-femto two-tier networks. The proposed method acquires the information on channel allocation and allows accessing of resource blocks to macrocells or femtocells users on demand basis. Chiranjibi Samal et al., in [39], have proposed a scheme based on FFR such that femtocells are permissible to selects those sub-bands which are not in utilize at macrocell, to mitigate interference in the LTE femtocell systems. In [40], authors present small-cells activation and sleeping mechanism, where cell-range expansion approach is used to covers the sleep cells that are far-off from macrocell and thereby macro BS serve users of sleeping cells in the vicinity. The presented mechanism confirms decrease in the energy consumption at each cell in heterogeneous networks. In [41], the spectrum wastage problem mainly occurs due to uneven distribution of users in a network is addressed and thus presented a scheme called contention-based FFR that improves the spectrum efficiency of cellular network to a great extend. In [42], both non-line of sight (NLOS) and line of sight (LOS) transmission is incorporate for analyzing performance of a HetCN by considering a user receiving high SINR from the BS. Authors in [43], incorporates both user association and enhanced Inter-Cell Interference Coordination (eICIC) management problems, and proposed a potential user association scheme that forward congested BSs users to adjacent BSs 
regardless of type. In [44], the Soft Frequency Reuse (SFR) performance with asymmetrical cell patterns in a realistic environment is presented, and provides an approach for efficient utilization of SFR in large networks with irregular cell patterns. In [45], authors proposed a scheme for efficient utilization and allocation of resource in OFDMA based femto-cell network, where macro cells are assigned frequencies using 3sectored FFR and femto cells are allocated with sub bands that are not used in macro cell area.

\section{Issues and Potentials}

In contrast to rural ones, radio propagation in urban areas is far complicated due to reflection, diffraction, blockage from buildings, obstacle environment etc. Accordingly, due to losses and attenuation in the received signal power, Macrocell BSs are facing problem in providing and maintaining of strong signals to indoor area users. In view of the fact that, today's lower carrier frequency will be replacing by higher one in next generation cellular system, so minimizing cell sizes and distance between BS and mobile users, can not only provide large capacity gain but also better coverage in indoors. A user deployed low power, low-cost small cells (micro, pico and femto BSs) provides not only wireless services but also enhances indoor coverage in cellular. Typically, Macrocell can contain hundreds of small BSs (e.g., femto-BSs for LTE networks), which provide indoor coverage of about ten meters and establishes a connection between core telephone network and users via wired internet. Consequently, micro, pico and femtocells are usually installed in poor coverage areas to seal the exposure gap of macrocell network. However, ICI in HetCNs is caused mainly due to random installation of small cells in macrocell area, and thereby results in overlapping of same frequency bands. Although small cells networks has promises to provide higher throughput with low cost but installation of small cells limits the transmit power of BS and resource utilization, and as a results use of FFR in uneven distribution of users may outcome wastage of spectrum in the network. In HetCNs, another challenge is to off-load the users from a macrocell to small cell BSs. Numerous researches have been observe related to interferences mostly associated with Macro-femto cells and has been solved up to some level. Some major issues such as Resource allocation and interference management, Cooperative communications, Co-Channel Interference and User association still need to be addressed and studied. To avoid interference, deployment of Macro-femto cells needs smart and efficient resource allocation schemes. Some adaptive approaches and techniques that have been proposed to mitigate interferences in multi-cellular heterogeneous networks includes FFR, Soft frequency reuse (SFR), Semi static FR method, Adaptive FR etc. Most well known techniques and approaches to combat the problem of user association are CellRange Expansion scheme, Utility-based BS selection method, enhanced ICIC scheme. Many of the BS selection methods deal with off-load of users from macro to small cells. In enhanced ICI scheme, macro BSs tends to mute data transmission in a periodic manner such that inter-tier interference can be minimized.

\section{Conclusion}

Heterogeneous cellular networks comprising small cells along with traditional macro towers are promising to provide high throughput, indoor services to users with low-cost etc for next generation cellular systems. These small cells e.g., metrocells, microcells, picocells, and femtocells along with distributed BSs offers advantages such as smartness, cheap, less obstructive, and easier installation compared to traditional homogeneous macrocell networks. In this paper, we present an outline of both traditional macrocell and heterogeneous network. The paper consist literature survey on recent and upcoming research areas associated with cellular network. Furthermore, some adaptive approaches and schemes that are proposed to combat problems of interferences, user association etc 
in cellular networks are discussed. The paper also discusses some relevant issues and potentials of deploying small cells along with macro cells to meet the expectations of users. Consequently, small cells overlaid with macro cells could be a favourable approach for OFDMA based next generation wireless networks such as WiMAX, LTE-Advanced etc.

\section{Acknowledgments}

The authors would like to thank Md. Anwar Hussain, Professor, Dept. of ECE, NERIST Deemed University, India for his valuable guidance and supports.

\section{References}

[1] T. S. Rappaport, "Wireless communications: principles and practice", New Jersey: Prentice Hall PTR, vol. 2, (1996).

[2] E. Hossain, D. In Kim and V. K. Bhargava, eds. Cooperative cellular wireless networks. Cambridge University Press, (2011).

[3] D. Tse and P. Viswanath, Fundamentals of Wireless Communication. Cambridge University Press, (2005).

[4] Md Hashem Ali Khan, Jin-Gyun Chung and Moon Ho Lee, "Downlink performance of cell edge using cooperative BS for multicell cellular network," EURASIP Journal on Wireless Communications and Networking, vol. 2016, no. 1, (2016), pp. 1-12.

[5] M. Abiri and M. Mehrjoo, "Improving the performance of cell edge users with fractional coordinated fair scheduling", IET Communications, vol. 8, no. 13, (2014), pp. 2315-2321.

[6] R. Ghaffar and R. Knopp, "Interference suppression strategy for cell-edge users in the downlink", IEEE Transactions on Wireless Communications, vol. 11, no. 1, (2012), pp. 154-165.

[7] X. You, D. Wang, P. Zhu and B. Sheng, "Cell edge performance of cellular mobile systems", IEEE Trans. on Selected Areas in Communications, vol. 29, no. 6, (2011), pp. 1139-1149.

[8] L. C. Wang and C-J Yeh, "3-cell network MIMO architectures with sectorization and fractional frequency reuse," IEEE Journal of Selected Areas in Communications, vol.29, no.6, (2011), pp.11851199.

[9] K. Hosseini, W. Yu and R. S. Adve, "Large-scale MIMO versus network MIMO for multicell interference mitigation”, IEEE Journal of Selected Topics in Signal Processing, vol. 8, no. 5, (2014), pp. 930-941.

[10] D. Gesbert, S. Hanly, H. Hung, S. S. Shitz, O. Simeone and W. Yu, "Multicell MIMO cooperative networks: a new look at interference", IEEE Journal of Selected Areas in Communications, vol. 28, no. 9, (2010).

[11] Y. Liang, A. Goldsmith and G. Foschini, "Evolution of base stations in cellular networks: denser deployment versus coordination," IEEE ICC, (2008), pp.4128-4132.

[12] H. Huang, M. Trivellato, A. Hottinen, M. Shafi, P. J. Smith, and R. Valenzuela, "Increasing downlink cellular throughput with limited network MIMO coordination”, IEEE Trans. Wireless Commun., vol. 8, no. 6, (2009), pp. 2983-2989.

[13] H. Huang, "Increasing throughput in cellular networks with higher-order sectorization", 2010 Conference Record of the Forty Fourth Asilomar Conference on Signals, Systems and Computers IEEE, (2010).

[14] I. Riedel and G. Fettweis, "Increasing throughput and fairness in the downlink of cellular systems with N-fold sectorization”, 2011 IEEE GLOBECOM Workshops (GC Wkshps), (2011).

[15] T. Novlan, J. G. Andrews, I. Sohn, R. K. Ganti and A. Ghosh, "Comparison of Fractional Frequency Reuse Approaches in the OFDMA Cellular Downlink", in IEEE Globecom 2010, (2010), pp. 1-5.

[16] S. H. Ali and V. C. M. Leung, "Dynamic frequency allocation in fractional frequency reused OFDMA networks", IEEE Transactions on Communications, vol. 8, no. 8, (2009).

[17] A. Najjar and N. Hamdi, "Efficient frequency reuse scheme for multi-cell OFDMA systems", IEEE ISCC, (2009), pp. 261-265.

[18] M. Assaad, "Optimal fractional frequency reuse (FFR) in multicellular OFDMA system", IEEE VTC Fall, (2008), pp. 1-5.

[19] Q. Li, "MIMO techniques in WiMAX and LTE: a feature overview", IEEE Communications magazine, vol. 48, no. 5, (2010), pp. 86-92.

[20] G. Boudreau, J. Panicker and N. Guo, "Interference coordination and cancellation for 4G networks", IEEE Commun. Mag., vol. 47, no. 4, (2009), pp. 74-81.

[21] Y. Li and Z. Zhang, "Co-channel interference suppression for multi-cell MIMO heterogeneous network", EURASIP Journal on Advances in Signal Processing, vol. 2016, no. 1, (2016), pp. 1-12.

[22] A. Thampi, "Mitigating inter-cluster interference on the uplink for a three-cell clustered cooperative network", EURASIP Journal on Wireless Communications and Networking, vol. 2016, no. 1, (2016). 
[23] X. Yongchao, "Co-channel interference suppression techniques for full duplex cellular system", China Communications, vol. 12, (2015), pp. 18-27.

[24] Y. Zhang, "Partial interference alignment for downlink multi-cell multi-input-multi-output networks", IET Communications, vol. 9, no. 6, (2015), pp. 836-843.

[25] D. Katselis, "On estimating the number of co-channel interferers in MIMO cellular systems", IEEE Signal Processing Letters, vol. 18, no. 6, (2011), pp. 379-382.

[26] G. Yu, D. Wen and F. Qu, "Joint user scheduling and channel allocation for cellular networks with full duplex base stations", IET Communications, vol. 10, no. 5, (2016), pp. 479-486.

[27] V. Sciancalepore, V. Mancuso and A. Banchs, "BASICS: Scheduling base stations to mitigate interferences in cellular networks", World of Wireless, Mobile and Multimedia Networks (WoWMoM), 2013 IEEE 14th International Symposium and Workshops, (2013).

[28] A. Asadi and V. Mancuso, "A survey on opportunistic scheduling in wireless communications", IEEE Communications Surveys \& Tutorials, vol. 15, no. 4, (2013), pp. 1671-1688.

[29] D. Lopez-Perez, X. Chu and J. Zhang, "Dynamic Downlink Frequency and Power Allocation in OFDMA Cellular Networks", IEEE Transactions on Communications, vol. 60, no. 10, (2012), pp. 2904-2914.

[30] D. Lopez-Perez, X. Chu, A. V. Vasilakos and H. Claussen, "Power Minimization Based Resource Allocation for Interference Mitigation in OFDMA Femtocell Networks", IEEE Journal on Selected Areas in Communications, vol. 32, no. 2, (2014), pp. 333-344.

[31] D. López-Pérez, X. Chu, A. V. Vasilakos and H. Claussen, "On Distributed and Coordinated Resource Allocation for Interference Mitigation in Self-Organizing LTE Networks", IEEE/ACM Transactions on Networking, vol. 21, no. 4, (2013), pp. 1145-1158.

[32] Y. Li, C. Niu, F. Ye and R. Q. Hu, "A universal frequency reuse scheme in LTE-A heterogeneous networks", Wirel. Commun. Mob. Comput., vol. 16, (2016), pp. 2839-2851.

[33] G. Huang and J. Li, "Interference Mitigation for Femtocell Networks Via Adaptive Frequency Reuse", in IEEE Transactions on Vehicular Technology, vol. 65, no. 4, (2016), pp. 2413-2423.

[34] A. Ebrahim and E. Alsusa, "Interference and Resource Management Through Sleep Mode Selection in Heterogeneous Networks", IEEE Transactions on Communications, vol. 65, no. 1, (2017), pp. 257-269.

[35] B. Zhuang, D. Guo and M. L. Honig, "Energy-Efficient Cell Activation, User Association, and Spectrum Allocation in Heterogeneous Networks", IEEE Journal on Selected Areas in Communications, vol. 34, no. 4, (2016), pp. 823-831.

[36] A. Saeed, E. Katranaras, M. Dianati and M. A. Imran, "Dynamic femtocell resource allocation for managing inter-tier interference in downlink of heterogeneous networks", IET Communications, vol. 10, no. 6, (2016), pp. 641-650.

[37] W. S. Jeon, J. Kim and D. G. Jeong, "Downlink Radio Resource Partitioning with Fractional Frequency Reuse in Femtocell Networks", IEEE Transactions on Vehicular Technology, vol. 63, no. 1, (2014), pp. 308-321.

[38] S. Rezvy, S. Rahman, A. Lasebae and J. Loo, "On demand-based frequency allocation to mitigate interference in Femto-Macro LTE cellular network", Second International Conference on Future Generation Communication Technologies (FGCT 2013), London, (2013), pp. 213-218.

[39] C. Samal, L. Samal and S. Das, "By Fractional Frequency Reuse mitigate interference in heterogeneous femto and macro cell networks", 2014 International Conference on Green Computing Communication and Electrical Engineering (ICGCCEE), Coimbatore, (2014), pp. 1-5.

[40] R. Tao, J. Zhang and X. Chu, "An Energy Saving Small Cell Sleeping Mechanism with Cell Expansion in Heterogeneous Networks", 2016 IEEE 83rd Vehicular Technology Conference (VTC Spring), Nanjing, (2016), pp. 1-5.

[41] H. Y. Zhan, B. J. Hu, Z. H. Wei, W. J. Liu, B. Li and X. Liu, "Contention-based fractional frequency reuse scheme in LTE/LTE-A network", 2016 IEEE Information Technology, Networking, Electronic and Automation Control Conference, Chongqing, (2016), pp. 663-666.

[42] B. Yang, G. Mao, X. Ge, H. H. Chen, T. Han and X. Zhang, "Coverage analysis of heterogeneous cellular networks in urban areas," 2016 IEEE International Conference on Communications (ICC), Kuala Lumpur, (2016), pp. 1-6.

[43] J. Choi, W. H. Lee, Y. Kim and S. C. Kim, "Dynamic user association and eICIC management in heterogeneous cellular networks," 2016 IEEE International Conference on Communications (ICC), Kuala Lumpur, (2016), pp. 1-6.

[44] L. Chen and D. Yuan, "Soft frequency reuse in large networks with irregular cell pattern: How much gain to expect?", 2009 IEEE 20th International Symposium on Personal, Indoor and Mobile Radio Communications, Tokyo, (2009), pp. 1467-1471.

[45] T. Lee, Jisun Yoon, Sangtae Lee and Jitae Shin, "Interference management in OFDMA Femtocell systems using Fractional Frequency Reuse", 2010 International Conference on Communications, Circuits and Systems (ICCCAS), Chengdu, (2010), pp. 176-180. 


\section{Authors}

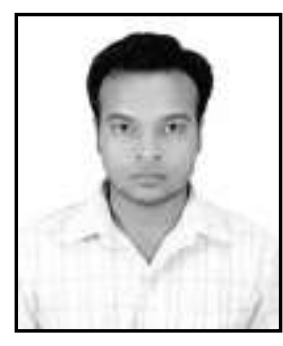

Janmoni Borah is currently pursuing $\mathrm{PhD}$ in Wireless Communication from NERIST Deemed University, India. He has received his B.Tech degree in ECE from CIT Kokrajhar, India in 2013 and M.Tech degree in Mobile Communication and Computing from NIT, Arunachal Pradesh, India in 2015. His research interest includes Wireless communication, Microstrip antenna design for multi-band applications, Microstrip filter design and Green computing. He has published some research papers in International journals and conferences.

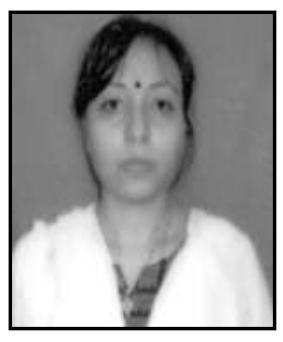

Joyatri Bora is currently working as an Assistant Professor in the Department of ECE at NERIST Deemed University, India. She did PhD in Electronics \& Communication Engineering from NERIST Deemed University, India in 2015. She received her B.Tech degree in Electrical Engineering from Jorhat Engineering College, India in 1995 and M.Tech degree in Electronics Design and Technology from Tezpur University, India in 2002. She has more than 10 years of experience in research and teaching field. She has published research articles in International referred journals and conferences. Her research interest includes Wireless Communication, Massive MIMO, and Bioelectronics. 
International Journal of Advanced Science and Technology Vol.109 (2017) 